\title{
Terapia de restricción-inducción de movimiento (CIMT), una técnica de utilidad para terapeutas ocupacionales que trabajan en neurorehabilitación
}

\author{
Castro González, Osvaldo
}

\section{Resumen}

Este artículo tiene por objetivo realizar una revisión de la terapia de restricción - inducción de movimiento (CIMT), técnica de tratamiento específica para personas que han sufrido un accidente cerebro vascular y que tienen como secuela una hemiparesia leve o moderada. Sus principios básicos incluyen la restricción del movimiento de la extremidad superior indemne durante un 90\% del día, y la estimulación del uso de la extremidad superior afectada en sesiones terapéuticas enfocadas prioritariamente a aspectos funcionales. Se mantiene este protocolo de entrenamiento durante doce días.

Durante este periodo de tratamiento se observan cambios a nivel cortical y funcional. Este artículo describe varios estudios que corroboran la eficiencia del programa, y que incluyen pruebas funcionales estandarizadas cuyos resultados se comparan con otras técnicas de tratamiento usadas en pacientes similares en el mismo periodo de tiempo.

Los Terapeutas Ocupacionales somos por esencia los profesionales del área de rehabilitación que buscamos que nuestros usuarios logren el máximo nivel de funcionalidad. Sin lugar a dudas, el conocer la existencia de técnicas como la CIMT, nos permitirá tener más herramientas para cumplir exitosamente este objetivo.

Palabras Clave: Accidente Cerebro Vascular, Hemiparesia, Neuroplasticidad, CIMT, Terapia Ocupacional, Rehabilitación.

\section{Abstract}

The main goal of this article is to review the Constraint-Induced Movement Therapy (C.I.M.T), a specific treatment technique employed for patients with stroke resulting in slight to moderate hemiparesia. Its basic principles include movement restriction of the unaffected upper extremity during $90 \%$ of the daily hours, and stimulating use of the affected upper extremity during therapy sessions with emphasis in functional aspects. This training protocol is maintained for 12 days.

During this treatment period, cortical and functional changes may be observed. This article describes various studies which confirm the program's efficiency, and that include standardized functional tests whose results are compared with other treatment techniques used in similar patients during the same time period.

Occupational Therapists are in essence health care professionals whose main concern is that our users obtain the highest level of functionality. With no doubt, the knowledge of techniques such as C.I.M.T. will give us the possibility to successfully accomplish this goal with a wider variety of tools.

Key Words: Stroke, Hemiparesia, Neuroplasticity, C.I.M.T, Occupational Therapy, Rehabilitation.

Terapeuta Ocupacional, Licenciado en Ciencias de la Ocupación Humana Universidad de Chile, Curso Básico de Neurodesarrollo Evaluación y Tratamiento en Hemiplejia de Adultos.(IBITA), Curso Avanzado de Neurodesarrollo en Evaluación y Tratamiento en Hemiplejia de Adultos (IBITA), Diploma en Neuropsicología Pontificia Universidad Católica de Chile, Magíster (c) en Terapia Ocupacional con mención en rehabilitación física, Universidad Andrés Bello, Terapeuta Ocupacional en Clínica Los Coihues, Profesor Universidad Autónoma de Chile, Profesor Universidad Nacional Andrés Bello. Contacto E-mail: ocgdocencia@gmail.com. Fono:465 7929 


\section{- Introducción .}

El Accidente cerebro vascular (ACV) constituye la tercera causa de muerte en la mayoría de los países desarrollados, después de la enfermedad coronaria y el cáncer y es una de las causas más importantes de morbilidad, con grandes implicancias económicas y familiares, debido a que la mayoría de las veces deja a la persona con secuelas que requerirán de un largo proceso de rehabilitación.

Los Terapeutas Ocupacionales que trabajamos en el área neurológica, recibimos un gran número de pacientes que han sufrido un ACV, al momento de plantear los objetivos de trabajo seleccionamos un marco teórico o bien combinamos algunos de estos, para que nos sirvan de hilo conductor en el proceso de rehabilitación.

Durante este proceso, mediante un entrenamiento intenso, se busca generar un aprendizaje motor, que induce la reorganización de la representación del movimiento y sinaptogénesis en la corteza motora del adulto, este aprendizaje motor no se desarrolla uniformemente durante las distintas sesiones de tratamiento, se caracteriza por una rápida fase inicial seguida por una segunda fase de aprendizaje mucho más lenta.

Es precisamente en esta etapa de aprendizaje lento del proceso de rehabilitación donde el Doctor Edward Taub, se concentró al desarrollar una innovadora terapia denominada "Constraint-induced movement therapy", la cual está protocolizada y basa en la evidencia su efectividad, ya que se han realizado numerosos estudios tanto con animales de laboratorio como en humanos, logrando corroborar mediante mapeos funcionales de la corteza cerebral, los cambios plásticos ocurridos en las distintas áreas del cerebro.

\section{- Accidente Cerebro Vascular y neuroplasticidad "}

El accidente cerebro vascular (ACV) concita renovado interés, tanto por su alto nivel de incidencia, mortalidad y carga secuelar, así como por los interesantes y esperanzadores avances en cuanto a terapias y modos de organización de los equipos de salud para su prevención y atención.

Según la American Stroke Association (ASA), en Estados Unidos, cada 45 segundos se presenta un episodio de ACV; y a su vez, cada 3 minutos ocurre una muerte por esta misma causa. La distribución para los diferentes tipos de ACV referidas a nuestro país es de un $88 \%$ para los accidentes cerebro vasculares de origen isquémico $(60 \%-70 \%$ aterotrombóticos y $10 \%-20 \%$ embólicos), y de un $12 \%$ para los de origen hemorrágico ( $9 \%$ intracerebrales y $3 \%$ subaracnoideas). ${ }^{(\underline{5})}$

En Chile, la ACV en su conjunto, consigna tasas de mortalidad, de 48,6 por 100.000 habitantes. En cuanto a demanda de atención de salud, la ACV constituiría el $6 \%$ de todas las hospitalizaciones de los adultos (excluidas las maternales), ocupando el segundo lugar como egreso hospitalario en los mayores de 65 años, considerada como la población de mayor riesgo. A pesar de todo lo anterior la tendencia evolutiva de la mortalidad de la ACV, es que ha ido disminuyendo. ${ }^{(5)}$ 
El impacto económico que suponen las ACV se explica por el alto porcentaje de secuelas (cerca de un 90\%) que deja en los pacientes que sufren esta patología, las que en muchos casos incapacitan al individuo, causando una demanda de cuidados y una necesidad de institucionalización con un gran gasto sanitario y social. ${ }^{(\underline{5})}$

Cabe destacar que cualquiera sea el origen del ACV, no causando la muerte del paciente éste provocará un daño en la zona directa del accidente, así como también puede causar daño parcial en zonas circundantes, las que se denominarán áreas de penumbra, en estas zonas no hay muerte neuronal, sino más bien enlentecimiento o déficit funcionales de las neuronas.

Las principales secuelas que puede dejar un accidente cerebro vascular son alteraciones conductuales, alteraciones en la percepción, alteraciones sensitivas, alteraciones cognitivas y alteraciones motoras.

Para mejorar estos déficit, generalmente se requiere de un abordaje multidiciplinario, preocupándose de trabajar cada área en forma específica y en forma conjunta a modo de reforzar constantemente cada uno de los avances.

Las alteraciones motoras van desde déficit en funciones tan básicas como control cefálico y control de tronco, hemiplejías o hemiparesias, es precisamente de este último grupo de pacientes que quedan con una hemiparesia post $A C V$ a quienes principalmente me referiré en el presente trabajo. ${ }^{(1)}$

Los marcos teóricos actuales hablan de una mayor recuperación motora al realizar una carga intensa de trabajo diario. Mediante ésta, lo que se busca es generar un aprendizaje motor ${ }^{(1)}$, que induce la reorganización de la representación del movimiento y sinaptogénesis de la corteza motora del adulto.

Esta habilidad motora no se desarrolla uniformemente durante las diferentes sesiones de tratamiento, caracterizándose por una rápida fase inicial, seguida de una segunda fase de aprendizaje motor mucho más lenta. El cómo la plasticidad cortical emerge en estas fases aún se desconoce. ${ }^{(\underline{3})}$

El aprendizaje es el proceso por el cual nosotros y otros animales adquirimos conocimientos sobre el mundo. La memoria es la retención o el almacenamiento de dicho conocimiento, incluso los animales con un sistema nervioso simple tienen la posibilidad de aprender de su ambiente, pero claramente esta capacidad alcanza su máxima expresión en los seres humanos.

Se ha dividido la memoria en aquella explícita, definida como la involucrada en el recuerdo consciente de información que se puede transformar y comunicar en palabras, y la memoria implícita, caracterizada por el recuerdo no consciente de capacidades como habilidades motoras (andar en bicicleta). La memoria explícita depende de la integridad del lóbulo temporal y de estructuras diencefálicas como el hipocampo, subiculum y corteza entorinal. La memoria implícita incluye formas simples de memoria asociativa como condicionamiento clásico, y no asociativas como la habituación, que depende de la integridad del cerebelo y ganglios básales. Aunque varias estructuras encefálicas juegan un rol en la consolidación de formas de aprendizaje y/o memoria, desde las observaciones realizadas en el paciente de 27 años conocido con las iniciales H.M. Con diagnostico de epilepsia refractaria, de foco en ambos lóbulos temporales. Quien fue sometido a extirpación temporal medial, donde 
sus hipocampos fueron removidos bilateralmente como tratamiento para su epilepsia, sus crisis disminuyeron notablemente, Sin embargo, H.M. Perdió la capacidad de almacenar información consciente por más de algunos segundos. Sólo podía aprender destrezas por repetición, aunque no se acordara de haberlo hecho. También podía recordar los eventos ocurridos previos a la intervención. Se ha reconocido que el hipocampo tiene un rol fundamental en la formación de la memoria declarativa, especialmente para síntesis de la memoria semántica y episódica. Se acepta ampliamente que la formación de la memoria es dependiente de los cambios en eficacia sináptica que permiten el refuerzo de asociaciones entre las neuronas; de hecho, la plasticidad sináptica actividad-dependiente, en las sinapsis apropiadas durante la formación de la memoria, se cree necesaria y suficiente a la vez, para el almacenaje de la información.

Ha sido difícil entender el mecanismo por el cual se puede alcanzar la consolidación de conexiones sinápticas, destacando en este esfuerzo el modelo de potenciación a largo plazo, relacionado a activación de receptores NMDA (N-metil-D-aspartato) y receptores metabotrópicos (receptores acoplados a proteínas $\mathrm{G}$ las cuales activan o desactivan la síntesis de segundos mensajeros) de glutamato.

Se pueden diferenciar por lo menos dos componentes de memoria: memoria de corto plazo, (de trabajo) de algunas horas de duración, y memoria a largo plazo, que persiste por varios días y a menudo mucho tiempo. En el nivel celular, el almacenaje de la memoria a largo plazo se asocia a la expresión de genes, síntesis de proteínas, y formación de nuevas conexiones sinápticas. ${ }^{(2)}$

Por su parte, por plasticidad cerebral o neuroplasticidad entenderemos la capacidad adaptativa del sistema nervioso para minimizar los efectos de las lesiones a través de modificar su propia organización estructural y funcional. ${ }^{(4)}$

Los fenómenos de plasticidad han sido estudiados mayormente en lesiones neurológicas no fatales, encontrándose varios tipos de clasificaciones de los cuales se describirán los más comúnmente utilizados y los mecanismos de plasticidad cerebral o neuroplasticidad más importantes: ${ }^{(\underline{6}, \underline{Z})}$

\section{Clasificación}

Por edades

a) Plasticidad del cerebro en desarrollo.

b) Plasticidad del cerebro en periodo de aprendizaje.

c) Plasticidad del cerebro adulto. ${ }^{(7)}$

Por patologías

a) Plasticidad del cerebro malformado.

b) Plasticidad del cerebro con enfermedad adquirida.

c) Plasticidad neuronal en las enfermedades metabólicas. (그) 
Por sistemas afectados

a) Plasticidad en las lesiones motrices.

b) Plasticidad en las lesiones que afectan cualquiera de los sistemas sensitivos.

c) Plasticidad en la afectación del lenguaje.

d) Plasticidad en las lesiones que alteran la inteligencia. $(\underline{7})$

\section{Mecanismos}

1. Ramificación o sinaptogénesis reactiva: crecimiento de un cuerpo celular hacia otro como consecuencia de su crecimiento normal. Un vacío en un sitio particular puede ser llenado parcialmente con la ramificación guiada por axones de crecimiento y proteínas como la laminina, integrina y cadherinas, con múltiples sitios de acoplamiento para neuronas, factores tróficos y glucoproteínas. Las ramificaciones colaterales son procesos axonales nuevos que han brotado de un axón no dañado y crecen hacia un sitio sináptico vacío.

Se ha demostrado que esto ocurre en el sistema nervioso central. Sin embargo, la ramificación puede ser adaptativa o maladaptativa, y su papel en la recuperación del daño cerebral es aún incierto.

2. Supersensibilidad de denervación: resulta de un permanente incremento de la respuesta neuronal por la disminución de las aferencias. El sitio receptor puede llegar a ser más sensible a un neurotransmisor o los receptores aumentar en número. Este podría ser un factor en la reorganización de sistema nervioso central.

3. Compensación conductual: después de un daño cerebral pueden desarrollarse nuevas combinaciones de conductas; un paciente puede usar diferentes grupos de músculos u otras estrategias cognoscitivas.

4. Neurotransmisión por difusión no sináptica: Este novel mecanismo se ha demostrado en pacientes con infarto cerebral; después de la destrucción de las vías dopaminérgicas existe incremento en la regulación de receptores de membrana extrasinápticos.

5. Desenmascaramiento: las conexiones neuronales en reposo que están inhibidas en el estado normal pueden desenmascararse después de un daño cerebral.

6. Factores tróficos: se relacionan con recuperación cerebral después de una lesión, además del factor de desarrollo nervioso, las integrinas, factor neurotrófico derivado del encéfalo, neurotrofina, factor neurotrófico ciliar, factor fibroblástico de desarrollo, factor neurotrófico derivado de la glia, etc.

7. Sinapsinas y neurotransmisores: las sinapsinas son fosfoproteínas que aglutinan vesículas sinápticas y las unen al citoesqueleto de las membranas. Los neurotransmisores además de mediar información transináptica pueden inducir efectos de sinaptogénesis y reestructuración neuronal. En otras formas de plasticidad sináptica, el calcio y otros mensajeros desencadenan eventos intracelulares, como la fosforilación proteica y los cambios en la expresión genética, que al final pueden conducir a cambios más permanentes en la potencia sináptica. 
8. Regeneración de fibras y células nerviosas: ocurre fundamentalmente en el sistema nervioso periférico, donde las células de Schwann proveen un ambiente favorable para los procesos de regeneración y facilitan la liberación de factores de desarrollo nervioso, factor neurotrófico derivado del encéfalo, neurotrofina.

9. Diasquisis: es un concepto antiguo que relaciona la recuperación de la función con la recuperación de la depresión neural desde sitios remotos, pero conectados al sitio de la lesión.

10. Neurotransmisores: se ha sugerido que algunos neurotransmisores se suman por medio de codificar información transináptica, lo cual induce efectos sobre la arquitectura neuronal, favoreciendo el desarrollo de retoños dendríticos, conexión de neuronas con influencias neuromoduladoras, entre otras.

11. Potenciación a largo plazo: este proceso cerebral de aprendizaje y memoria que involucra la plasticidad sináptica ha centrado su campo experimental en estudios sobre la transmisión del glutamato y del receptor N-metil-D-aspartato (NMDA). Lo relevante de la información científica es que la consolidación de los códigos y procesos de memoria en lo mamíferos están relacionados con estímulos de potenciación a largo plazo. $\stackrel{(8,9)}{(2)}$

\section{- Terapia de restricción inducción de movimiento y neuroplasticidad "}

La Terapia de restricción-inducción de movimiento (Constraint-Induced Movement Therapy, o en forma abreviada, CIMT) es una técnica de rehabilitación desarrollada por Edward Taub, Ph.D. y colaboradores. Comienza con estudios experimentales en el año 1977 en primates, a los cuales se les producía una desaferentación quirúrgica (Rizotomia dorsal) provocando de esta manera, un daño neurológico que daba como secuela una hemiparesia en una de sus extremidades superiores. ${ }^{(3)}$ Los primates dejaron inmediatamente de usar la extremidad afectada y no generaban ningún tipo de actividad espontánea con ella.

Sin embargo, el uso de la extremidad desaferentada podía ser inducido mediante la inmovilización del brazo intacto y el entrenamiento de la extremidad afectada. De esta forma, se logró reutilizar el brazo afectado, cuya utilidad fue permanente, manteniéndose durante el resto de la vida del primate.

Estas evidencias experimentales indican que la pérdida de la función motora por desaferentación fue el resultado de un aprendizaje de un comportamiento limitado denominado por Taub "aprendizaje del no uso". Este mismo mecanismo fue pensado para ser aplicado en humanos que sufren de una hemiparesia leve a moderada posterior a un $\mathrm{ACV} . \underline{(3)}$

La investigación de esta técnica en humanos, ha alcanzado sus mayores logros en términos de evidencias en las ultimas dos décadas, Miltner y cols. y Van Deer Lee y cols. $\stackrel{(10,11)}{ }$ Investigaron en forma diferenciada la utilidad de esta terapia en pacientes secuelados de un ACV en su etapa crónica (hasta nueve años después del ACV) utilizando, ambos estudios, pacientes que ya habían recibido terapias físicas en su etapa aguda y subaguda, y que tenían algún grado de movilidad en muñeca ( $20^{\circ}$ de 
extensión de muñeca) y mano ( $10^{\circ}$ de extensión de la articulación metacarpofalángica (MCF), la intervención consistió en la restricción de la movilidad de la extremidad superior no afectada poniéndole un mitón por un tiempo que correspondiera al $90 \%$ que el paciente se mantuviera despierto por un periodo de 12 días, y el entrenamiento de la extremidad superior afectada por cerca de siete horas diarias, durante los doce días hábiles que duró el estudio.

Este entrenamiento consistió en una técnica denominada "Shaping", la cual se enfoca en la realización de tareas funcionales fraccionadas y con un incremento paulatino de la dificultad.

Los resultados de ambos estudios demostraron que la CIMT tiene efectos positivamente significativos en la rehabilitación de pacientes crónicos post ACV, medidos por escalas funcionales y comparados con otros tipos de terapias como la terapia bimanual, se demuestra además un efecto mantenido en el tiempo de las destrezas obtenidas con la terapia y que los mayores logros se alcanzaron con pacientes con alteraciones sensitivas.

El hecho de que los logros se mantengan seis meses posterior a la aplicación de la terapia indica que la CIMT actúa como un fuerte estímulo, capaz de incrementar la reorganización cortical, mediante un tratamiento intenso de ejercicios funcionales de destreza manual, generando cambios plásticos a nivel central.

Además, ambos estudios aportan un importante descubrimiento, ya que al lograr recuperación funcional en pacientes secuelados de ACV de larga data, contradicen los conceptos clínicos que se tenían hasta la fecha, los cuales planteaban que los logros terapéuticos funcionales de la extremidad superior parética son sólo posibles dentro del primer año post ACV. $(\underline{12})$

\section{- Terapia de restricción-Inducción de movimiento Modificada (Modified Costraint-Induced Movement Therapy o en forma abreviada, mCIMT) .}

A pesar de la eficacia demostrada por la CIMT, se ha cuestionado su implementación dentro de centros clínicos, considerándola limitada y cuestionándole principalmente la intensidad y duración de las sesiones. Se realizaron estudios que indicaban el malestar de un alto porcentaje de pacientes que no estaban dispuestos a seguir un régimen tan intenso, pero sí preferían un protocolo de mayor duración y con entrenamientos más cortos, disminuyendo también el tiempo de restricción de uso de la extremidad no afectada. $\left.{ }^{(13}\right)$

Fue esta situación la que generó la creación de una terapia que mantuviera los principios del CIMT, pero con nuevas características que la pudieran hacer más grata y accesible para los pacientes, de esta manera se creó la $M C I M T$, como alternativa para ser implementada dentro de un plan de tratamiento y con la esperanza de tener una mejor aceptación de los pacientes.

La mCIMT utiliza los mismos principios de la CIMT, pero con un aumento en la duración del protocolo, el cual pasó de dos a 10 semanas, con 30 minutos de Kinesiología enfocada a entrenamiento de posición bípeda, marcha y 30 minutos de Terapia 
Ocupacional enfocada a la ejecución de tares funcionales con la extremidad superior afectada. Se restringió durante estas 10 semanas el uso de la extremidad superior indemne, cinco días a la semana durante cinco horas estando el paciente despierto.

Se realizaron dos estudios para medir la eficacia de esta técnica, uno en pacientes agudos $^{(13)}$ y uno en pacientes sub-agudos. ${ }^{(14)}$

En los estudios se obtuvieron resultados similares, evidenciándose logros terapéuticos importantes en el estudio con pacientes agudos $\left.{ }^{(13}\right)$ y mejoras funcionales estadísticamente significativas en el estudio con pacientes sub-agudos ${ }^{(\underline{14})}$ comparándolos con un grupo control al cual se le aplicaron terapias tradicionales como técnica de facilitación neuromuscular propioceptiva ${ }^{(14)}$ que fueron medidos con pautas de evaluación estandarizadas como The fugl-meyer assessment of motor recovery after stroke (FMA) que evalúa la dimensión de los déficit de la extremidad superior, la Action Research Arm (ARA) que valora la capacidad de destreza con la extremidad superior y The Motor Activity Log (Mal) que mide cómo los pacientes utilizan su extremidad superior afectada en las actividades de la vida diaria en el hogar.

Se determinó con estas pautas de forma cuantificable que mCIMT, genera un aumento significativo en las habilidades y destrezas de la extremidad afectada.

\section{Conclusiones}

Las investigaciones realizadas por Taub en primates nos revelan la existencia de reorganización cortical o neuroplasticidad posterior a una injuria o daño en el sistema nervioso. Posterior a la ocurrencia de un accidente cerebro vascular ocurre un complejo patrón de reorganización cortical en las distintas etapas de este cuadro, durante la etapa sub-aguda ocurre una disminución en la excitabilidad de la corteza motora y una reducción de la representación cortical de los músculos paréticos, lo cual podría deberse al daño neuronal estructural o al desuso de la extremidad superior afectada o ambos procesos en forma simultánea.

Sin embargo, hasta antes de la existencia de la CIMT, no existían hallazgos basados en la evidencia que reflejaran que en la etapa crónica post ACV, podían ocurrir cambios plásticos en la corteza o reorganización cortical.

Debido a la alta prevalencia que presentan los ACV, a nivel mundial y nacional, es necesario implementar cambios tanto en la actitud y organización de los planes de trabajo para atender a los pacientes secuelados Post ACV.

Nosotros como Terapeutas Ocupacionales tenemos un rol fundamental en la recuperación funcional de nuestros usuarios con hemiplejía, si contamos con la evidencia científica que existe reorganización cortical o neuroplasticidad, incluso en periodos de cronicidad de la enfermedad y si sabemos que muchas de las actividades cotidianas que realizamos, en las distintas áreas de desempeño ocupacional, requieren de actividad bimanual, deberíamos probar la utilización de técnicas como la descrita en este articulo, antes de aplicar en forma prematura técnicas compensatorias, como el cambio de lateralidad o uso de ayudas técnicas. 


\section{- Referencias}

(1) Carr J, Shepard R, Rehabilitación en pacientes en el ictus, Sección I, Editorial Elsevier, 2004; 3-33.

(2) Kandel E, Jessel T, Schwartz J, Principios de Neurociencia, seccion VI y IX, Editorial Mcgraw-hill . Interamericana, 2001. 653-872; 1169-1279.

(3) Taub E, Uswatte G, Elvert T. March 2002. New treatment in neurorehabilitatión founded on basis research, revista Nature (Review), volume 3:228-236.

(4) Galaburda AM. Introduction to special issue: Developmental plasticity and recovery of function. Neuropsychologia 1990;28:515-516.

(5) Instituto Nacional de Estadística. 1995. Seguimiento de las diez causas principales de muerte compatibilizadas en 1960, 1965, 1970, 1975, 1985, 1990 y 1995 En: INE, editor. Anuario Demográfico. Santiago.

(6) Escobar ML, Bermudez-Rattoni F. Long-term potentiation in the insular cortex enhances conditioned taste aversion retention. Brain Res 2000;852: 208-212.

(7) Bach-y-Rita P, Wicab-Bach-y-Rita E. Biological and psychological factors in recovery from brain damage in humans. Can J Psychol 1990;44:148-165.

(8) Fingers S, Wolft C. The Kennard principle before Kennard. The early history of age and brain lesions. Arch Neurol 1988;45:1136-1142.

(9) Buchwald JS. Comparison of plasticity in sensory and cognitive processing system. Clin Perinat 1990;17:57-66.

(10) Miltner W, Bauder H, Sommer M, Pysch D, Dettmers C, Taub E. Effects of Contraint-Induced Movement Therapy on Patients With Chronic Motor Deficits after stroke. Stroke 1999; 30: 586-592.

(11) Van Der Lee J, Wagenaar R, Lankhorts G, Vogelaar T, Deville W, Bouter L, Forced Use of the Upper Extremity in Chronic Stroke Patients, Stroke 1999; 30: $2396-2375$. 
(12) Gerald B, Smith P, Keneth H, Silver M, Andrew P, Goldberg M "Task-Oriented" exercice Improve Hamstring Strength and Spastic reflexes in Chronic Stroke Patients. Stroke, 1999; 30: 2112-2118.

(13) Page S, Sisto S, Levine P, Modified Constrain-Induced Therapy, in Chronic Stroke. Am J. Phys. Med Rehabil. 2002; 81: 870-875.

(14) Page S, Sisto S, Jhonston M, Levine P, Huges M. Modified Constrain-Induced Movement Therapy in Subagute Stroke.: A Case Report. Arch Phys Med.

Rehabil. 2002; 83: 286-290.

\section{Agradecimientos:}

A mi colega Anne Marie Bonnefoy, de Clinica Los Coihues, por su valioso aporte en la traducción del abstract. 